\title{
在 $\mathrm{Pt} / \mathrm{SrTiO}_{3}$ 衬底上制备高 $c$ 轴取向 $\mathrm{PbTiO}_{3}$ 薄膜*
}

卢朝请 蒋昌忠 (1) 王世敏 石 瑛(1) 黄桂玉 赵建洪顾豪爽仰安祥 (湖北大学物理系, 武汉 430062; (1)武汉大学物理系, 武汉 430072)

\section{关链词 $\mathrm{PbTiO}$, 薄膜、c 轴取向、sol-gel 方法、Pt/SrTiO, 村底}

铁电 $\mathrm{PbTiO}_{3}$ 薄膜是一种优良的热释电材料. 特别是 $c$ 轴取向的 $\mathrm{PbTiO}_{3}$ 薄谟, 因为 $c$ 轴就是极化轴, 并且 $c$ 轴取向的 $\varepsilon_{r}$ 低 (约为 100 ), 所以它的热释电系数大,介电常数小, 热容 量小, 很有希望成为高性能指数, 高灵敏度的红外传感器材料. 国外用珲射法在 $\mathrm{MgO}$ (100) 单晶和外延的 $\mathrm{Pt}$ 膜上制备了高 $c$ 轴取向的 $\mathrm{PbTiO}_{3}$ 薄膜, 并报道了其优良的电学性质 ${ }^{[1]}$.

新近发展起来的 sol-gel 方法与溅射法相比, 制备铁电薄膜有组分易控制, 设备简单, 成 本低, 易于工业化生产, 成膜面积大且均匀, 热处理温度低等优点. Chen 等 ${ }^{[2]}$ 首次报道了用 sol-gel 方法在 $\mathrm{MgO}$ (100) 单晶上生长 $c$ 轴取向的 $\mathrm{PbTiO}_{3}$ 薄膜. 但是目前还未见报道用 sol-gel 方法制得的 $c$ 轴取向 $\mathrm{PbTiO}_{3}$ 薄膜的电学性质. 我们曾报道用 sol-gel 方法在 $\mathrm{SrTiO}_{3}(100)$ 单晶基片上制备高 $c$ 轴取向的 $\mathrm{PbTiO}_{3}$ 薄膜 ${ }^{[3]}$. 本文介绍用贼 射法在 $\mathrm{SrTiO}_{3}$ (100) 单晶上制备高取向的 Pt $(100)$ 薄膜, 再在 Pt 膜上用 sol-gel 方法制备高 $c$ 轴取向的 $\mathrm{PbTiO}_{3}$ 薄膜, 并测量了高取向 $\mathrm{PbTiO}_{3}$ 薄膜的介电常数.

由于 $\mathrm{SrTiO}_{3}$ 衬底本身不导电, 为了能测量 $c$ 轴取向 $\mathrm{PbTiO}_{3}$ 薄膜的电学性质, 就要给薄 膜设计一层下表面电极薄膜, 并这层电极薄膜必须是外延或高取向的, 为了保证 $\mathrm{PbTiO}_{3}$ 薄膜 在其上仍能沿 $c$ 轴取向生长. 实验证明选用 $\mathrm{Pt}$ 电极材料是理想的, 这是因为不仅 $\mathrm{Pt}$ 的晶体 结构、晶格常数和热膨胀系数与 $\mathrm{SrTiO}_{3}$ 和 $\mathrm{PbTiO}_{3}$ 较匹配,而且 $\mathrm{Pt}$ 的导电性好, 附着力强, 化学稳定性好, 耐高温退火.

\section{1 实 验}

在自制的三枪直流磁控溅射装置上用平面磁控贼射法在 $\mathrm{SrTiO}_{3}(100)$ 单晶上制备 $\mathrm{Pt}$ 薄膜. 溅射靶材是 $\varnothing 40 \mathrm{~mm}$ 的高纯铂片, 靶与基片距离 $40 \mathrm{~mm}$, 践射条件如表 1 .

用 sol-gel 方法在 $\mathrm{Pt}(100) / \mathrm{SrTiO}_{3}(100)$ 衬底上制备高 $c$ 轴取向 $\mathrm{PbTiO}_{3}$ 薄膜的过程同 文献[3]. $\mathrm{Pt}$ 膜和 $\mathrm{PbTiO}_{3}$ 薄膜的物相和晶粒取向分析在日本理学 $\mathrm{D} / \mathrm{max}-\mathrm{IIIC}$ 型 X 射线衍 射仪上进行, $\mathrm{CuK} \alpha$ 辐射, 管压 $40 \mathrm{kV}$, 管流 $25 \mathrm{~mA}, \mathrm{DS}, \mathrm{RS}$ 和 $S S$ 狭輅分别是 $1^{\circ}, 0.3 \mathrm{~mm}$ 和 $1^{\circ}$, 扫描速率 $2 \% \mathrm{~min}$. 薄膜厚度用 SEM 观察衬底的横断面测量.

用蒸镀法给 $\mathrm{PbTiO}_{3}$ 取向薄膜上表面披一层 $\mathrm{Al}$ 电极, 电极尺寸 $\varnothing 1.5 \mathrm{~mm}$. 用 $4192 \mathrm{~A}$ 型 低频阻抗分析仪测量其介电常数. 
表 1 制备 Pt(100) 高取向薄膜的践射条件

\begin{tabular}{c|c|c|c|c|c}
\hline $\begin{array}{c}\text { 残射气体 } \\
(\%)\end{array}$ & $\begin{array}{c}\text { 践射气压 } \\
(\mathrm{Pa})\end{array}$ & $\begin{array}{c}\text { 基片温度 } \\
\left({ }^{\circ} \mathrm{C}\right)\end{array}$ & $\begin{array}{c}\text { 沉积速率 } \\
(\mathrm{nm} / \mathrm{min})\end{array}$ & $\begin{array}{c}\text { 弧压 } \\
(\mathrm{V})\end{array}$ & $\begin{array}{c}\text { 弧流 } \\
(\mathrm{mA})\end{array}$ \\
\hline $\mathrm{Ar}(80)+\mathrm{O}_{2}(20)$ & 0.5 & 600 & 10 & 420 & 10 \\
\hline
\end{tabular}

\section{2 结果与讨论}

\section{1 践射法制备 Pt(100) 取向薄膜}

用溅射法制备的 Pt 膜表面光洁. 图 1 中 (a) 和 (b) 分别是按表 1 践射条件在石英玻璃 上制备的无择优取向的 Pt 膜和在 $\mathrm{SrTiO}_{3}$ (100) 单晶上得到的沿(100)高取向 Pt 膜的 XRD 图,膜厚 $0.2 \mu \mathrm{m}$. 可以看出, Pt 膜没有 (100) 和 (300) 衍射峰; 曲线 (b) 中除 $\operatorname{Pt}(200)$ 峰与 $\mathrm{SrTiO}_{3}(200)$ 峰重叠外, 仅有一个弱的 $\mathrm{Pt}(111)$ 峰. 为了肯定 $\mathrm{Pt}(200)$ 峰的存在和强度, 在 制膜前给 $\mathrm{SrTiO}_{3}$ 基片作了 XRD 分析, 其 XRD 图如图 1(c), 衍射仪的使用条件不变. 很 明显,戝射一层 $\mathrm{Pt}$ 膜后, $\mathrm{SrTiO}_{3}$ 的 (100)和(300)峰变弱, 而在 $2 \theta$ 约 $46.49^{\circ}$ 处的衍射峰增强 了; 这是因为 $\mathrm{Pt}(200)$ 面对 $\mathrm{X}$ 光子的散射能力比 $\mathrm{SrTiO}_{3}(200)$ 面强. 可见, $\mathrm{Pt}(200)$ 峰的确 存在,且它的强度比 $\mathrm{Pt}(111)$ 峰强得多.事实上,下一步能在 $\mathrm{Pt}$ 膜上生长 $c$ 轴取向的 $\mathrm{PbTiO}_{3}$ 薄膜,也从另一个侧面证明了 Pt 膜的(100)高取向性.

实验表明,基片温度、沉积速率、贼射气体、浌射气压和浌射时间等因素对 Pt 膜的取向和 质量都有较大影响. 小的沉积速率有利于 $\mathrm{Pt}$ 膜的高取向生长. 浌射时间太短, Pt 薄膜根本

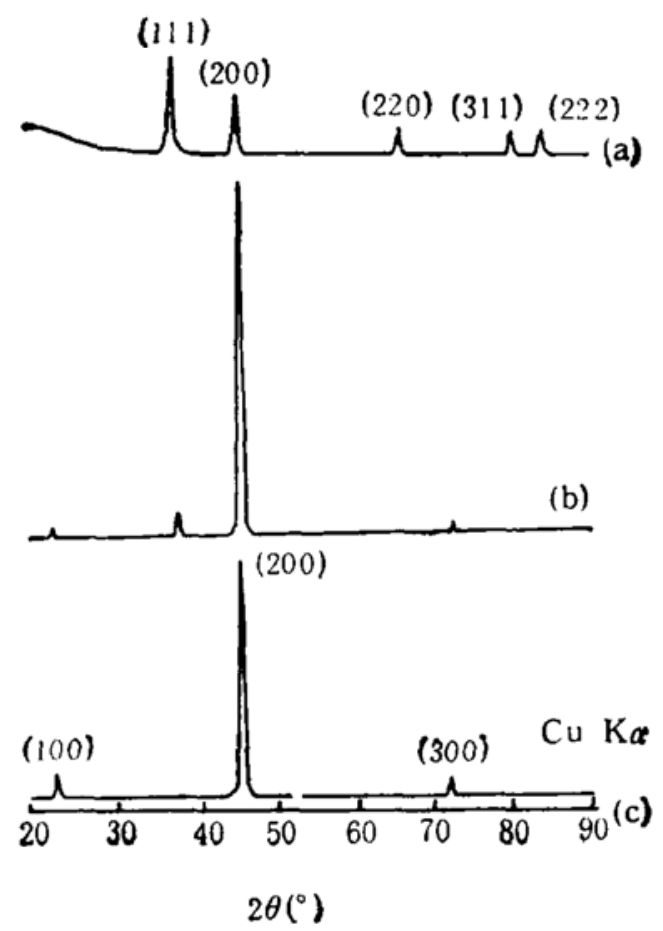

图 $1 \mathrm{Pt}$ 薄膜和 $\mathrm{SrTiO}$, 基片的 XRD 图 (a) $\mathrm{Pt} /$ 石英玻璃, (b) $\mathrm{Pt} / \mathrm{SrTiO}_{3}(100)$, (c) $\mathrm{SrTiO}_{3}(100)$ 单晶基片
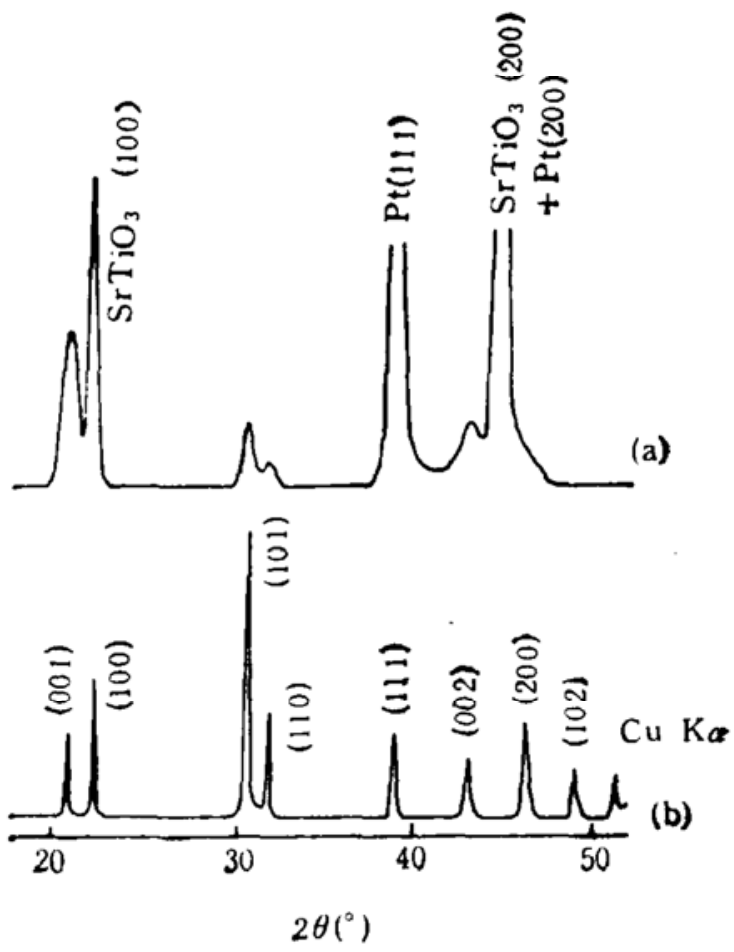

图 $2 \mathrm{PbTiO}_{3} / \mathrm{Pt} / \mathrm{SrTiO}_{3}$ 薄膜 (a) 和 $\mathrm{PbTiO}$, 粉末的 XRD 图 (b) 
不导电, 说明 Pt 膜还没有形成连续结构; 贼射时间太长又会降低 Pt 膜的 $(100)$ 取向度.

\section{2 离 $\mathrm{c}$ 轴取向 $\mathrm{PbTiO}_{3}$ 薄膜}

把 $0.4 \mathrm{~mol} / \mathrm{L}$ 浓度的溶胶多次重复匀胶在 $\mathrm{Pt}(100) / \mathrm{Sr}^{\mathrm{T}} \mathrm{TiO}_{3}(100)$ 衬底上, 缓慢升温至 $600^{\circ} \mathrm{C}$ 保温足够长时, 然后随炉缓缓降至室温, 制备了厚 $0.5 \mu \mathrm{m}$ 的 $\mathrm{PbTiO}_{3}$ 薄膜. 薄膜呈浅 黄绿色透明状,表面光洁如镜, 其 XRD 图如图 2(a) 所示. 为了弄清是否有 $\mathrm{PbTiO}_{3}(100)$ 峰与 $\mathrm{SrTiO}_{3}(100)$ 峰重叠在一起, 对 $\mathrm{PbTiO}_{3}$ 薄膜样品在 $\mathrm{SrTiO}_{3}$ 的 $(200),(300)$ 和 (400)等 高 $2 \theta$ 角衍射峰处作了 X 射线阶梯慢扫描, 未发现有 $\mathrm{PbTiO}_{3}$ 的 $(n 00)$ 峰从 $\mathrm{SrTiO}_{3}(n 00)$ 峰 中分离出来; 说明的确没有 $\mathrm{PbTiO}_{3}(100)$ 峰,即使有, 它的强度也是很弱. 仔细观察还可看到 $\mathrm{SrTiO}_{3}(100)$ 峰比 $\mathrm{PbTiO}_{3}(001)$ 峰尖锐得多, 这也可说明上述结论, 因为 $\mathrm{PbTiO}_{3}$ 薄膜 (100) 衍射峰应与它的(001) 峰一样都有晶粒尺寸致宽. 图 2(b) 是用 sol-gel 方法制备的 $\mathrm{PbTiO}_{3}$ 细粉的 XRD 图,有很多衍射峰出现, 是典型的 $\mathrm{PbTiO}_{3}$ 多晶谱. 比较 $\mathrm{PbTiO}_{3}$ 薄膜和粉末的 $\mathrm{XRD}$ 图可以看出, $\mathrm{PbTiO}_{3}$ 薄膜的(101)峰和(110)峰相对变弱了许多, 而 $(001)$ 和 $(002)$ 峰增 强了不少; 说明 $\mathrm{PbTiO}_{3}$ 薄膜是沿 $c$ 轴择优取向的,其取向度

$$
P=\frac{3.2 I(001)}{3.2 I(001)+I(101)}=90 \% ，
$$

这里系数 3.2 是无择优取向 $\mathrm{PbTiO}_{3}$ 多晶的 $I(101) / I(001)$ 比值.

大量实验摸索发现, $\mathrm{PbTiO}_{3}$ 薄膜的 $c$ 轴取向度强烈依赖于 $\mathrm{SrTiO}_{3}(100)$ 单晶的质量和 $\mathrm{Pt}$ 膜的 (100) 取向度; 低的降温速率对提高 $\mathrm{PbTiO}_{3}$ 薄膜的 $c$ 轴取向度有利, 快速降温不仅可 能导致 $\mathrm{PbTiO}_{3}$ 薄膜开裂, 而且会大大降低 膜的取向度. 我们认为, $\mathrm{PbTiO}_{3}$. 薄膜能沿 $c$ 轴取向, 主要是因为薄膜从烧结温度降至室 温的过程中在居里点附近发生的结构 相变, 以及因薄膜和基底的热膨胀系数不同导致薄 膜内产生的压应力引起. 也正因为应力普遍 存在于薄膜之中, 我们没能制得 $\mathrm{PbTiO}_{3}$ 单 晶薄膜.

图 3 是薄膜和衬底的横断面的 SEM 照 片. 可见, $\mathrm{PbTiO}_{3}$ 薄膜和 $\mathrm{Pt}$ 电极薄膜的厚

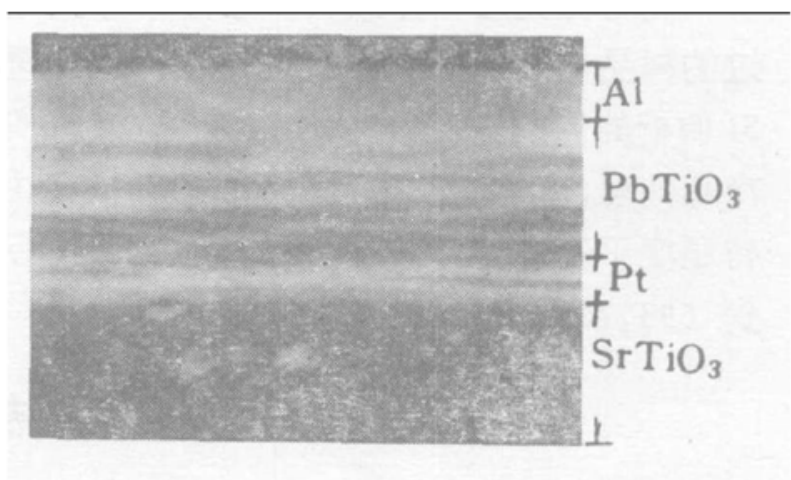

图 3 上电极 $\mathrm{Al} / \mathrm{PbTiO}$, 澺膜/ $\mathrm{Pt}$ 电极海膜/ $\mathrm{SrTiO}_{3}$ 祄底横断面的 SEM 照片 度均匀, 二者附着紧密. $\mathrm{PbTiO}_{3}$ 薄膜的电阻率达 $10^{12} \Omega \mathrm{cm}$ 以上. 击穿场强大于 $150 \mathrm{kV} / \mathrm{cm}$, 说明薄膜内没有裂纹和针孔. 室温下, $100 \mathrm{kHz}$ 频率时薄膜的相对介电常数 $\varepsilon_{\mathrm{s}}$ 为 82 , 比 $\mathrm{PbTiO}_{3}$ 晶体 $c$ 轴方向的 $\varepsilon_{\mathrm{r}}(126)$ 低 ${ }^{[4]}$, 这是因为薄膜的表面层有较低的介电常数. 有关高 $a$ 轴取向 $\mathrm{PbTiO}_{3}$ 薄膜的介电、铁电和热释电等性质的详细结果将另文报道.

致谢衰心感谢武汉大学物理系范湘军教授和郭怀喜副教授的大力支持和协作。

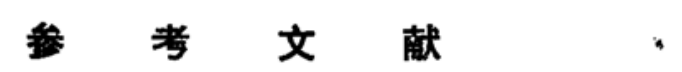

[1] Iijima, K., Tomita, Y., Takayama, R. et al., J. Appl. Phys., 1986, 60(1): 361-367.

[2] Chen, C., Phder, D. F., J. Amer. Ceram. Soc., 1989, 72: 1495-1499.

[3] 顾豪爽、们安样、包定华等,科学通报，1992,37(17): 1561-1562.

[4] lijima, K., Kawashima, S., Ueda, I., J pn. J. A ppl. Phys., 1985, 24(2): 482-486. 